\title{
SOME RENEWAL THEOREMS WITH APPLICATION TO A FIRST PASSAGE PROBLEM
}

\author{
By C. C. Heyde \\ Michigan State University
}

Reprint2d from The Annals of Mathematical Statistics

Vol. 37, No. 3, June, 1966

Printed in U.S.A. 


\title{
SOME RENEWAL THEOREMS WITH APPLICATION TO A FIRST PASSAGE PROBLEM ${ }^{1}$
}

\author{
By C. C. Heyde
}

\section{Michigan State University}

1. Introduction. Let $X_{i}, i=1,2,3, \cdots$ be a sequence of independent and identically distributed random variables with $E\left|X_{i}\right|<\infty, E X_{i}=\mu>0$. Write $X_{i}^{-}=-\min \left(0, X_{i}\right), S_{n}=\sum_{i=1}^{n} X_{i}$ and $M_{n}=\max _{1 \leqq k \leqq n} S_{k}$. In this paper we shall discuss the asymptotic behaviour as $x \rightarrow \infty$ of the sums $\sum_{n=1}^{\infty} a_{n} \operatorname{Pr}\left(S_{n} \leqq x\right)$ and $\sum_{n=1}^{\infty} a_{n} \operatorname{Pr}\left(M_{n} \leqq x\right)$ for certain classes of positive coefficient sequences $\left\{a_{n}\right\}$ and use the results on the latter sums to investigate the behaviour of the first passage time out of the interval $(-\infty, x]$ for the process $S_{n}$ as $x \rightarrow \infty$.

The analysis that we shall use in obtaining the theorems on asymptotic behaviour follows closely on that of Smith [6] who discussed sums $\sum_{n=1}^{\infty} a_{n} \operatorname{Pr}\left(S_{n} \leqq x\right)$ for a class of coefficient sequences that we shall also discuss and for non-identically distributed random variables. In fact, our Theorem 1 follows directly from a specialization of the analysis of Smith. One of the particularly interesting characteristics of this technique is that it enables us to study the asymptotic behaviour of the sums $\sum_{n=1}^{\infty} a_{n} \operatorname{Pr}\left(S_{n} \leqq x\right)$ and $\sum_{n=1}^{\infty} a_{n} \operatorname{Pr}\left(M_{n} \leqq x\right)$ in the one operation in spite of essential differences in their behaviour.

2. Renewal theorems. For the first set of positive term coefficient sequences $\left\{a_{n}\right\}$ that we consider we shall suppose (as in [6]) that there exist real numbers $\alpha>0, \gamma \geqq 0$ and some non-negative function of slow growth $L(x)$ such that

$$
\sum_{n=1}^{\infty} a_{n} x^{n} \sim\left[\alpha /(1-x)^{\gamma}\right] L(1-x)^{-1}, \quad \text { as } \quad x \rightarrow 1^{-} .
$$

This is satisfied, for example, if

$$
a_{n} \sim[\alpha / \Gamma(\gamma)] n^{\gamma-1} L(n) \quad \text { as } \quad n \rightarrow \infty
$$

using an Abelian theorem of Doetsch [3], 460.

In the subsequent work we shall need the following definition:

Definition. The index $k$ of the sequence $\left\{a_{n}\right\}$ is the least real $k$ such that $a_{n}=O\left(n^{k}\right)$.

Consideration will be restricted to cases where $\sum a_{n}$ diverges.

Theorem 1. Suppose $E|X|<\infty, E X=\mu>0$. Let $k$ be the index of the sequence $\left\{a_{n}\right\}$ and $m$ be non-negative. In order that

$$
\sum_{n=1}^{\infty} a_{n} \operatorname{Pr}\left(S_{n} \leqq x\right) \sim[\alpha L(x) / \Gamma(1+\gamma)](x / \mu)^{\gamma} \quad \text { as } \quad x \rightarrow \infty
$$

for each sequence $\left\{a_{n}\right\}$ such that $k \leqq m$ it is necessary and sufficient that $E\left|X^{-}\right|^{m+2}<\infty$.

Received 2 June 1965; revised 6 January 1966.

${ }^{1}$ Research supported in part by the National Institutes of Health. 
Theorem 2. Suppose $E|X|<\infty, E X=\mu>0$. Let $k$ be the index of the sequence $\left\{a_{n}\right\}$ and $m$ be non-negative. In order that

$$
\sum_{n=1}^{\infty} a_{n} \operatorname{Pr}\left(M_{n} \leqq x\right) \sim[\alpha L(x) / \Gamma(1+\gamma)](x / \mu)^{\gamma} \quad \text { as } \quad x \rightarrow \infty
$$

for each sequence $\left\{a_{n}\right\}$ such that $k \leqq m$ it is necessary that $E\left|X^{-}\right|^{m+1}<\infty$ and sufficient that $E\left|X^{-}\right|^{m+2}<\infty$.

I conjecture that the condition $E\left|X^{-}\right|^{m+1}<\infty$ is both necessary and sufficient in Theorem 2. It is certainly known that in the particular case where $a_{n}=1$ for all $n$ so that $k=0$ we only need $E\left|X^{-}\right|<\infty$ (see for example Chow and Robbins [2]).

The proof of the two theorems shall be deferred until we have given four lemmas. The first of these is given in a form which is more general than we shall need subsequently as it has some independent interest.

Lemma 1. Suppose the random variables $X_{i}, i=1,2,3, \cdots$ are independent and identically distributed. Write $S_{n}=\sum_{i=1}^{n} X_{i}, M_{n}=\max _{1 \leqq k \leqq n} S_{k}$, and, in the case where $E\left|X_{i}\right|<\infty, E X_{i}=\mu$. If $E\left|X_{i}\right|^{r}<\infty$ with $1 \leqq r<2$ and $\mu \geqq 0$, then

$$
n^{-1 / r}\left(M_{n}-n \mu\right) \rightarrow_{\text {a.s. }} 0 .
$$

If $E\left|X_{i}\right|^{r}<\infty$ with $0<r<1$ or $E\left|X_{i}\right|^{r}<\infty$ with $1 \leqq r<2$ and $\mu<0$, then

$$
n^{-1 / r} M_{n} \rightarrow \text { a.s. } 0 .
$$

("a.s." denotes almost sure convergence).

The corresponding almost sure convergence versions for the sums $S_{n}$ have been given by Kolmogorov $(r=1)$ and Marcinkiewicz $(r \neq 1)$ (see for example Loève [5] 242, 243).

Proof. Suppose $E\left|X_{i}\right|^{r}<\infty$. Let $c_{r}=\mu$ if $1 \leqq r<2 ; c_{r}=0$ if $0<r<1$. If $c_{r} \geqq 0$, we have

$$
\begin{aligned}
M_{n}-n c_{r} & =\max _{1 \leqq j \leqq n} S_{j}-n c_{r} \\
& \leqq \max _{1 \leqq j \leqq n}\left(S_{j}-j c_{r}\right) \\
& \leqq \max _{1 \leqq j \leqq n} j^{1 / r} a_{j},
\end{aligned}
$$

where $a_{j}=j^{-1 / r}\left|S_{j}-j c_{r}\right|$. Therefore, if $M_{n} \geqq n c_{r}, 0 \leqq M_{n}-n c_{r} \leqq$ $\max _{1 \leqq j \leqq n} j^{1 / r} a_{j}$, and if $M_{n}<n c_{r}, 0>M_{n}-n c_{r} \geqq S_{n}-n c_{r}=-n^{1 / r} a_{n} \geqq$ $-\max _{1 \leqq j \leqq n} a_{j}$, so that $0 \leqq n^{-1 / r}\left|M_{n}-n c_{r}\right| \leqq \max _{1 \leqq j \leqq n} n^{-1 / r} j^{1 / r} a_{j}$. In order to obtain the desired result in this case it suffices to show that for arbitrary $\epsilon>0$,

$$
\operatorname{Pr}\left(\mathbf{U}_{k \geqq n}\left\{\max _{1 \leqq j \leqq k} j^{1 / r} k^{-1 / r} a_{j} \geqq \epsilon\right\}\right) \rightarrow 0 \text { as } n \rightarrow \infty .
$$

Now the strong laws of Kolmogorov and Marcinkiewicz ([5], 242, 243) imply that for arbitrary $\epsilon>0$,

$$
\operatorname{Pr}\left(\max _{j \geqq n} a_{j} \geqq \epsilon\right) \rightarrow 0 \quad \text { as } \quad n \rightarrow \infty .
$$

Therefore, given $\eta>0$ arbitrarily small we can choose an $N$ so large that

$$
\operatorname{Pr}\left(\max _{j>N} a_{j} \geqq \frac{1}{2} \epsilon\right) \leqq \frac{1}{2} \eta
$$


and then select an $n_{0}(>N)$ so large that for $n \geqq n_{0}$,

$$
\operatorname{Pr}\left(N^{1 / r} n^{-1 / r} \max _{1 \leqq j \leqq N} a_{j} \geqq \frac{1}{2} \epsilon\right) \leqq \frac{1}{2} \eta .
$$

Then, for $n \geqq n_{0}$,

$$
\begin{aligned}
\operatorname{Pr}\left(\mathbf{U}_{k \geqq n}\{\right. & \left.\left.\max _{1 \leqq j \leqq k} j^{1 / r} k^{-1 / r} a_{j} \geqq \epsilon\right\}\right) \\
& =\operatorname{Pr}\left(\bigcup_{k \geqq n}\left\{\max _{1 \leqq j \leqq N} j^{1 / r} k^{-1 / r} a_{j}+\max _{N<j \leqq k} j^{1 / r} k^{-1 / r} a_{j} \geqq \epsilon\right\}\right) \\
& \leqq \operatorname{Pr}\left(\bigcup_{k \geqq n}\left\{\max _{1 \leqq j \leqq N} j^{1 / r} k^{-1 / r} a_{j}+\max _{N<j \leqq k} a_{j} \geqq \epsilon\right\}\right) \\
& \leqq \operatorname{Pr}\left(N^{1 / r} n^{-1 / r} \max _{1 \leqq j \leqq N} a_{j}+\max _{j>N} a_{j} \geqq \epsilon\right) \\
& \leqq \operatorname{Pr}\left(N^{1 / r} n^{-1 / r} \max _{1 \leqq j \leqq N} a_{j} \geqq \frac{1}{2} \epsilon\right)+\operatorname{Pr}\left(\max _{j>N} a_{j} \geqq \frac{1}{2} \epsilon\right) \\
& \leqq \eta,
\end{aligned}
$$

using (2) and (3). This completes the proof of this part of the lemma. It remains to consider the case $c_{r}<0$.

In the case $c_{r}<0, M_{n}^{+}=\max \left(0, M_{n}\right)$ actually has a proper limiting distribution (finite with probability one), $M^{+}=\lim _{n \rightarrow \infty} M_{n}{ }^{+}$.

We have

$$
\begin{aligned}
& \operatorname{Pr}\left(\mathrm{U}_{k \geqq n}\left\{k^{-1 / r}\left|M_{k}\right| \geqq \epsilon\right\}\right) \\
& =\operatorname{Pr}\left(\mathrm{U}_{k \geqq n}\left\{k^{-1 / r} M_{k} \geqq \epsilon\right\} \cup \mathbf{U}_{k \geqq n}\left\{k^{-1 / r} M_{k} \leqq-\epsilon\right\}\right) \\
& \leqq \operatorname{Pr}\left(\mathbf{U}_{k \geqq n}\left\{k^{-1 / r} M_{k} \geqq \epsilon\right\}\right)+\operatorname{Pr}\left(\mathbf{U}_{k \geqq n}\left\{k^{-1 / r} M_{k} \leqq-\epsilon\right\}\right) \\
& \leqq \operatorname{Pr}\left(\mathbf{U}_{k \geqq n}\left\{M^{+} \geqq k^{1 / r} \epsilon\right\}\right)+\operatorname{Pr}\left(\mathbf{U}_{k \geqq n}\left\{X_{1} \leqq-k^{1 / r} \epsilon\right\}\right) \\
& =\operatorname{Pr}\left(M^{+} \geqq n^{1 / r} \epsilon\right)+\operatorname{Pr}\left(X_{1} \leqq-n^{1 / r} \epsilon\right)
\end{aligned}
$$

and both these terms approach zero as $n \rightarrow \infty$. Therefore, $n^{-1 / r} M_{n} \rightarrow_{\text {a.s. }} 0$ as required. This completes the proof of the lemma.

In the subsequent work we shall write $F_{n}(x)=\operatorname{Pr}\left(S_{n} \leqq x\right), G_{n}(x)=$ $\operatorname{Pr}\left(M_{n} \leqq x\right)$ and $H_{n}(x)$ to mean either $F_{n}(x)$ or $G_{n}(x)$ (so that if a property holds for both $F_{n}(x)$ and $G_{n}(x)$ it holds for $H_{n}(x)$ and conversely).

Lemma 2. $\int_{\mu}^{\infty}\left\{1-H_{n}(n x)\right\} d x \rightarrow 0$ as $n \rightarrow \infty$.

Proof. Introduce the new random variables $Y_{i}=\max \left(0, X_{i}\right), i=1,2,3, \cdots$. Suppose $E Y_{i}=\alpha$. If $M_{n}>x$ then $\sum_{i=1}^{n} Y_{i}>x$ so that $\operatorname{Pr}\left(\sum_{i=1}^{n} Y_{i}>x\right) \geqq$ $\operatorname{Pr}\left(M_{n}>x\right) \geqq \operatorname{Pr}\left(S_{n}>x\right)$ and hence

$$
1-F_{n}(x) \leqq 1-G_{n}(x) \leqq 1-K_{n}(x),
$$

where we have written $K_{n}(x)=\operatorname{Pr}\left(\sum_{i=1}^{n} Y_{i} \leqq x\right)$.

We show firstly that $\int_{\alpha}^{\infty}\left\{1-K_{n}(n x)\right\} d x \rightarrow 0$ as $n \rightarrow \infty$. We have, by a simple integration by parts,

$$
\begin{aligned}
\alpha & =\int_{0}^{\infty}\left\{1-K_{n}(n x)\right\} d x \\
& =\int_{0}^{\alpha}\left\{1-K_{n}(n x)\right\} d x+\int_{\alpha}^{\infty}\left\{1-K_{n}(n x)\right\} d x .
\end{aligned}
$$

Further, by the law of large numbers, $K_{n}(n x) \rightarrow 0$ as $n \rightarrow \infty$ for $x<\alpha$. It follows 
from the mean value theorem that $\int_{0}^{\alpha}\left\{1-K_{n}(n x)\right\} d x \rightarrow \alpha$ as $n \rightarrow \infty$ and hence that $\int_{\alpha}^{\infty}\left\{1-K_{n}(n x)\right\} d x \rightarrow 0$ as $n \rightarrow \infty$.

Then, making use of $(4)$,

$$
\begin{aligned}
0 \leqq \int_{\mu}^{\infty}\left\{1-F_{n}(n x)\right\} d x & \leqq \int_{\mu}^{\infty}\left\{1-G_{n}(n x)\right\} d x \\
& \leqq \int_{\mu}^{\alpha}\left\{1-G_{n}(n x)\right\} d x+\int_{\alpha}^{\infty}\left\{1-K_{n}(n x)\right\} d x .
\end{aligned}
$$

Now, in view of Lemma 1 (case $r=1, \mu>0$ ), $G_{n}(n x) \rightarrow 1$ as $n \rightarrow \infty$ for $x>\mu$ so that by the mean value theorem $\int_{\mu}^{\alpha}\left\{1-G_{n}(n x)\right\} d x \rightarrow 0$ as $n \rightarrow \infty$. We have shown that $\int_{\alpha}^{\infty}\left\{1-K_{n}(n x)\right\} d x \rightarrow 0$ as $n \rightarrow \infty$ so the proof is complete.

Lemma 3. (Smith [6]). If the non-negative constants $\left\{a_{n}\right\}$ satisfy (1) then as $s \rightarrow 0+$,

$$
\sum_{n=1}^{\infty} a_{n} e^{-\mu s n} \sim \alpha(\mu s)^{-\gamma} L\left(s^{-1}\right) .
$$

Lemma 4. If the non-negative constants $\left\{a_{n}\right\}$ satisfy (1) then as $s \rightarrow 0+$,

$$
\begin{gathered}
\sum_{n=1}^{\infty} n a_{n} e^{-\mu s n} \sim \alpha(\mu s)^{-\gamma-1} L\left(s^{-1}\right) \quad \text { if } \quad \gamma>0 \\
\lim \sup _{s \rightarrow 0+} s\left[L\left(s^{-1}\right)\right]^{-1} \sum_{n=1}^{\infty} n a_{n} e^{-\mu s n}<\alpha \mu^{-1} \text { if } \gamma=0 .
\end{gathered}
$$

Smith [6] has established the former result, ignoring the possibility of the case $\gamma=0$. The result given above for $\gamma=0$ can be readily extracted from Smith's proof and is adequate in the present context.

Proof of Theorems 1 and 2. We follow the methods of [6] but work the proof in terms of $H_{n}(x)$.

Suppose firstly that $E\left|X^{-}\right|^{m+2}<\infty$. Take $\beta$ arbitrary with $0<\beta<\mu$. Consider

$$
\begin{aligned}
0 \leqq K_{n} & =\int_{n \beta}^{n \mu} e^{-s x} H_{n}(x) d x \quad(s \geqq 0) \\
& =n \int_{\beta}^{\mu} e^{-n s x} H_{n}(n x) d x \\
& \leqq n e^{-n \beta s} \int_{\beta}^{\mu} H_{n}(n x) d x .
\end{aligned}
$$

Now using the law of large numbers and the inequality $H_{n}(y) \leqq F_{n}(y)$ (or alternatively referring to Lemma 1 as well), we see that $H_{n}(n x) \rightarrow 0$ as $n \rightarrow \infty$ for all $x<\mu$. Hence, using the mean value theorem, we may write

$$
K_{n}=n e^{-n \beta s} \delta_{n}{ }^{\prime},
$$

where $\delta_{n}{ }^{\prime} \rightarrow 0$ as $n \rightarrow \infty$, uniformly in $s \geqq 0$.

Now consider

$$
\begin{aligned}
0 \leqq L_{n} & =\int_{n \mu}^{\infty} e^{-s x}\left\{1-H_{n}(x)\right\} d x \\
& =n \int_{\mu}^{\infty} e^{-n s x}\left\{1-H_{n}(n x)\right\} d x \\
& \leqq n e^{-n s \beta} \int_{\mu}^{\infty}\left\{1-H_{n}(n x)\right\} d x .
\end{aligned}
$$$$
(s \geqq 0)
$$

In view of Lemma 2 , we may write

$$
L_{n}=n e^{-n \beta s} \delta_{n}^{\prime \prime}
$$

where $\delta_{n}^{\prime \prime} \rightarrow 0$ as $n \rightarrow \infty$, uniformly in $s \geqq 0$. 
Combining (5) and (6) and putting $\delta_{n}=\delta_{n}{ }^{\prime}-\delta_{n}{ }^{\prime \prime}$, we obtain

$$
\sum_{n=1}^{\infty} a_{n}\left(L_{n}-K_{n}\right)=-\sum_{n=1}^{\infty} n a_{n} \delta_{n} e^{-n \beta s} .
$$

Now given arbitrary $\epsilon>0$ we can choose an integer $n_{0}(\epsilon)$ so large that $\left|\delta_{n}\right|<\epsilon$ for all $n>n_{0}$. Then,

$$
\left|\sum_{n=1}^{\infty} n a_{n} \delta_{n} e^{-n \beta s}\right|<\sum_{n=1}^{n_{0}} n a_{n}\left|\delta_{n}\right| e^{-n \beta s}+\epsilon \sum_{n=1}^{\infty} n a_{n} e^{-n \beta s},
$$

so that by Lemma 4 ,

$$
\begin{aligned}
\lim \sup _{s \rightarrow 0+}\left[(\mu s)^{\gamma+1} / L\left(s^{-1}\right)\right]\left|\sum_{n=1}^{\infty} n a_{n} \delta_{n} e^{-n \beta s}\right| & \leqq \epsilon \gamma \alpha \text { if } \gamma>0 \\
& \leqq \epsilon \alpha \text { if } \gamma=0
\end{aligned}
$$

and hence

$$
\left[(\mu s)^{\gamma+1} / L\left(s^{-1}\right)\right] \sum_{n=1}^{\infty} a_{n}\left(L_{n}-K_{n}\right) \rightarrow 0 \quad \text { as } \quad s \rightarrow 0+.
$$

Next write

$$
\begin{aligned}
\Phi_{\beta}(x) & =\sum_{n=1}^{\infty} a_{n} H_{n}(x) U(x-n \beta) \\
& =\sum_{n=1}^{\infty} a_{n} U(x-n \mu)-\sum_{n=1}^{\infty} a_{n}\left\{U(x-n \mu)-H_{n}(x)\right\} U(x-n \beta)
\end{aligned}
$$

where

$$
\begin{aligned}
U(x) & =1, \quad x \geqq 0, \\
& =0, \quad x<0 .
\end{aligned}
$$

If we denote the Laplace transform of a function $A(x)$ by $A^{0}(s)=\int_{0}^{\infty} e^{-s x} A(x) d x$ we have

$$
\Phi_{\beta}^{0}(s)=s^{-1} \sum_{n=1}^{\infty} a_{n} e^{-n \mu s}-\sum_{n=1}^{\infty} a_{n}\left(L_{n}-K_{n}\right)
$$

the term by term integration being justified by monotone convergence.

From (8), (10) and Lemma 3 it follows that

$$
\left[(\mu s)^{\gamma+1} / L\left(s^{-1}\right)\right] \Phi_{\beta}^{0}(s) \rightarrow \alpha \mu \quad \text { as } \quad s \rightarrow 0+.
$$

Appealing to a Tauberian theorem of Doetsch [3], 511, we then obtain

$$
\left[1 / t^{\gamma+1} L(t)\right] \int_{0}^{t} \Phi_{\beta}(x) d x \rightarrow \alpha / \mu^{\gamma} \Gamma(\gamma+2) .
$$

Now for $t>0$ and $0<\theta<1$,

$$
\Phi_{\beta}(\theta t)(t-\theta t) \leqq \int_{\theta t}^{t} \Phi_{\beta}(x) d x \leqq \Phi_{\beta}(t)(t-\theta t)
$$

so that

$\left[1 / t^{\gamma} L(t)\right] \Phi_{\beta}(\theta t) \leqq[1 /(1-\theta)]\left\{\left[1 / t^{\gamma+1} L(t)\right] \int_{0}^{t} \Phi_{\beta}(x) d x\right.$

$$
\left.-\left[1 / t^{\gamma+1} L(t)\right] \int_{0}^{\theta t} \Phi_{\beta}(x) d x\right\} \leqq\left[1 / t^{\gamma} L(t)\right] \Phi_{\beta}(t) .
$$

Then, for fixed $\theta$ and $t \rightarrow \infty,\left[1 / t^{\gamma+1} L(t)\right] \int_{0}^{t} \Phi_{\beta}(x) d x \rightarrow \alpha / \mu^{\gamma} \Gamma(\gamma+2)$, $\left[1 /(\theta t)^{\gamma+1} L(t)\right] \int_{0}^{\theta t} \Phi_{\beta}(x) d x \rightarrow\left[1 /(\theta t)^{\gamma+1} L(\theta t)\right] \int_{0}^{\theta t} \Phi_{\beta}(x) d x \rightarrow \alpha / \mu^{\gamma} \Gamma(\gamma+2)$ by 
(12) so that

(13) $\lim \sup _{t \rightarrow \infty}\left[1 / t^{\gamma} L(t)\right] \Phi_{\beta}(\theta t) \leqq\left[\alpha / \mu^{\gamma} \Gamma(\gamma+2)\right]\left(1-\theta^{\gamma+1}\right) /(1-\theta)$

$$
\leqq \lim \inf _{t \rightarrow \infty}\left[1 / t^{\gamma} L(t)\right] \Phi_{\beta}(t) .
$$

Taking $\theta \rightarrow 1$ in the right hand part of inequality (13) gives

$$
\lim \inf _{t \rightarrow \infty}\left[1 / t^{\gamma} L(t)\right] \Phi_{\beta}(t) \geqq \alpha / \mu^{\gamma} \Gamma(\gamma+1) .
$$

Further, the left hand part of the inequality (13) can be written $\lim \sup _{t \rightarrow \infty}\left[1 /(\theta t)^{\gamma} L(\theta t)\right] \Phi_{\beta}(\theta t) \leqq\left[-\theta \alpha / \mu^{\gamma} \Gamma(\gamma+2)\right]\left(1-\theta^{-(\gamma+1)}\right) /(1-\theta)$ and the left hand side of this is equal to $\lim \sup _{t \rightarrow \infty}\left[1 / t^{\gamma} L(t)\right] \Phi_{\beta}(t)$ for $\theta$ fixed. Then, taking $\theta \rightarrow 1$ in the right hand side, we obtain

$$
\lim \sup _{t \rightarrow \infty}\left[1 / t^{\gamma} L(t)\right] \Phi_{\beta}(t) \leqq \alpha / \mu^{\gamma} \Gamma(\gamma+1),
$$

so that combining (14) and (15),

$$
\lim _{t \rightarrow \infty}\left[1 / t^{\gamma} L(t)\right] \Phi_{\beta}(t)=\alpha / \mu^{\gamma} \Gamma(\gamma+1) .
$$

Now under the condition $E\left|X^{-}\right|^{m+2}<\infty$ we certainly have for all $x,-\infty<$ $x<\infty, \sum n^{m} H_{n}(x)<\infty$ in view of Theorem A of Heyde [4] and the inequality $H_{n}(x) \leqq F_{n}(x)$. Hence, $\sum a_{n} H_{n}(x)<\infty$, since the sequence $\left\{a_{n}\right\}$ has index $k \leqq m$. Write

$$
\sum_{n=1}^{\infty} a_{n} H_{n}(x)=\Phi_{\beta}(x)+\Psi_{\beta}(x)
$$

where

$$
\Psi_{\beta}(x)=\sum_{n=1}^{\infty} a_{n} H_{n}(x)\{1-U(x-n \beta)\} .
$$

We shall go on to show that $\Psi_{\beta}(x) / x^{\gamma} L(x) \rightarrow 0$ as $x \rightarrow \infty$.

Define a new sequence of random variables $Y_{i}, i=1,2,3, \cdots$ by

$$
Y_{i}=X_{i}-\beta \text {. }
$$

Then, $E Y>0$ and $E\left|Y^{-}\right|^{m+2}<\infty$ since $E\left|X^{-}\right|^{m+2}<\infty$. It follows from Theorem A of [4] applied to the $Y$ 's that for $k \leqq m, \sum n^{k} F_{n}(n \beta)<\infty$, and since $H_{n}(n \beta) \leqq$ $F_{n}(n \beta)$,

$$
\sum n^{k} H_{n}(n \beta)<\infty .
$$

Also, it is clear from (18) that $\Psi_{\beta}(x) \leqq \sum_{n=1}^{\infty} a_{n} H_{n}(n \beta)$ and (19) ensures that this upper bound is finite since $k$ is the index of the sequence $\left\{a_{n}\right\}$. We therefore have $\Psi_{\beta}(x) / x^{\gamma} L(x) \rightarrow 0$ as $x \rightarrow \infty$ and hence, using (16) and (17),

$$
\sum_{n=1}^{\infty} a_{n} H_{n}(x) \sim[\alpha L(x) / \Gamma(1+\gamma)](x / \mu)^{\gamma} \quad \text { as } \quad x \rightarrow \infty .
$$

This result is true for all sequences $\left\{a_{n}\right\}$ with index $k \leqq m$ and hence establishes the sufficiency parts of both Theorems 1 and 2 .

The necessity parts of both the theorems are easy to establish. It suffices to 
note that in particular $\sum n^{m} H_{n}(x)<\infty$ for all $x,-\infty<x<\infty$, and hence by Theorem A of [4] in the case $H_{n}(x)=F_{n}(x)$ we obtain $E\left|X^{-}\right|^{m+2}<\infty$ and by Theorem A of [4] together with the well known inequality $G_{n}(0)=\operatorname{Pr}\left(M_{n} \leqq 0\right)$ $\geqq n^{-1} \operatorname{Pr}\left(S_{n} \leqq 0\right)$ in the case $H_{n}(x)=G_{n}(x)$ we obtain $E\left|X^{-}\right|^{m+1}<\infty$. This completes the proof of both Theorems 1 and 2 .

Some remarks on the possibility that the condition $E\left|X^{-}\right|^{m+1}<\infty$ might be both necessary and sufficient in Theorem 2 would seem in order. To establish that this is the case, it would be adequate to show that if $E X=\mu>0$ and $E\left|X^{-}\right|^{k+1}<\infty$, then for $0<\beta<\mu$,

$$
\sum n^{k} G_{n}(n \beta)=\sum n^{k} \operatorname{Pr}\left(M_{n} \leqq n \beta\right)<\infty .
$$

The nearest approach to this that I have obtained is summarized in the following theorem:

Theorem 3. Suppose $E|X|<\infty, E X>0, \operatorname{Pr}(X<0)>0$, and let $k$ be a non-negative integer. A necessary and sufficient condition for the convergence of the series

$$
\sum_{n=1}^{\infty} n^{k} \operatorname{Pr}\left(M_{n} \leqq x\right), \quad-\infty<x<\infty,
$$

is that $E\left|X^{-}\right|^{k+1}<\infty$.

Proof. It follows from the work of Heyde [4] that a necessary and sufficient condition for the convergence of the series $\sum n^{k} \operatorname{Pr}\left(M_{n} \leqq 0\right)$ is that $E\left|X^{-}\right|^{k+1}<\infty$. Therefore, in order to complete the proof it is only necessary to show that the convergence of $\sum n^{k} \operatorname{Pr}\left(M_{n} \leqq 0\right)$ implies that of $\sum n^{k} \operatorname{Pr}\left(M_{n} \leqq x\right)$, $0<x<\infty$.

To accomplish this we define a new sequence of random variables:

$$
\begin{aligned}
& N_{0}=S_{0}=0 \\
& N_{1}=\max \left(S_{0}, S_{1}\right)=X_{1}^{+}, \\
& N_{2}=\max \left(S_{0}, S_{1}, S_{2}\right)=\left(X_{1}+{X_{2}^{+}}^{+},\right. \\
& \cdots \\
& N_{n}=\max \left(S_{0}, S_{1}, S_{2}, \cdots, S_{n}\right)=\left(X_{1}+\left(X_{2}+\cdots+\left(X_{n-1}+X_{n}^{+}\right)^{+} \cdots\right)^{+}\right. \\
& \cdots
\end{aligned}
$$

Since the $X_{i}$ are independent and identically distributed we may write $N_{0}=0$, $N_{n+1} \sim\left(X+N_{n}\right)^{+}, n \geqq 1$, where for two random variables $X$ and $Y$, we write $X \sim Y$ if they have the same distribution. Clearly, $N_{n}=M_{n}^{+}=\max \left(0, M_{n}\right)$, so that

$$
\begin{aligned}
& \operatorname{Pr}\left(N_{n} \leqq x\right)=\operatorname{Pr}\left(M_{n} \leqq x\right), \quad x>0, \\
& \operatorname{Pr}\left(N_{n}=0\right)=\operatorname{Pr}\left(M_{n} \leqq 0\right) .
\end{aligned}
$$

Now for $A>0, n \geqq 1$, we have

$$
\operatorname{Pr}\left(M_{n+1} \leqq A\right)=\int_{-\infty}^{A} \operatorname{Pr}\left(M_{n} \leqq A-y\right) d \operatorname{Pr}(X \leqq y)
$$


so that for arbitrary $B>0$,

$$
\begin{aligned}
\sum_{n=1}^{N} n^{\alpha} \operatorname{Pr}\left(M_{n+1} \leqq A\right) & =\int_{-\infty}^{A}\left\{\sum_{n=1}^{N} n^{\alpha} \operatorname{Pr}\left(M_{n} \leqq A-y\right)\right\} d \operatorname{Pr}(X \leqq y) \\
\geqq & \int_{-\infty}^{-B}\left\{\sum_{n=1}^{N} n^{\alpha} \operatorname{Pr}\left(M_{n} \leqq A-y\right)\right\} d \operatorname{Pr}(X \leqq y) \\
& \geqq\left\{\sum_{n=1}^{N} n^{\alpha} \operatorname{Pr}\left(M_{n} \leqq A+B\right)\right\} \operatorname{Pr}(X \leqq-B) .
\end{aligned}
$$

Then, choosing $B$ so that $\operatorname{Pr}(X \leqq-B)>0$, we see that if $\sum n^{\alpha} \operatorname{Pr}\left(M_{n} \leqq A\right)$ converges then $\sum n^{\alpha} \operatorname{Pr}\left(M_{n} \leqq A+B\right)$ converges. Let $A \rightarrow 0+$; we see that the convergence of $\sum n^{\alpha} \operatorname{Pr}\left(M_{n} \leqq 0\right)$ implies that of $\sum n^{\alpha} \operatorname{Pr}\left(M_{n} \leqq r B\right)$ for all positive integral $r$ and hence that of $\sum n^{\alpha} \operatorname{Pr}\left(M_{n} \leqq x\right)$ for all $x$, $0<x<\infty$. This completes the proof of the theorem.

The restriction to non-negative integral $k$ in Theorem 3 is unfortunate. This comes about from the use of the derivatives of the generating function

$$
\sum_{n=0}^{\infty} \operatorname{Pr}\left(M_{n} \leqq 0\right) t^{n}=\exp \left\{\sum_{n=1}^{\infty} n^{-1} t^{n} \operatorname{Pr}\left(S_{n} \leqq 0\right)\right\}
$$

in [4].

We now go on to consider coefficient sequences of the form $a_{n}=e^{r n}, r>0$, and shall establish the following two theorems:

Theorem 4. Suppose $E|X|<\infty, E X=\mu>0$. In order that

$$
\sum_{n=1}^{\infty} e^{r n} \operatorname{Pr}\left(S_{n} \leqq x\right) \sim r^{-1} e^{x r \mu-1} \quad \text { as } \quad x \rightarrow \infty
$$

for any $r$ in some interval $0<r<R$, it is necessary and sufficient that $X^{-}$should have an analytic characteristic function.

(The term "analytic characteristic function" is used for a characteristic function which is analytic in a strip containing the origin as an interior point.)

Theorem 5. Suppose $E|X|<\infty, E X=\mu>0$. In order that

$$
\sum_{n=1}^{\infty} e^{r n} \operatorname{Pr}\left(M_{n} \leqq x\right) \sim r^{-1} e^{x r \mu-1} \quad \text { as } \quad x \rightarrow \infty
$$

for any $r$ in some interval $0<r<R$, it is necessary and sufficient that $X^{-}$should have an analytic characteristic function.

The proofs of these theorems, of course, follow markedly similar lines to the proofs of Theorems 1 and 2. A generalized coefficient sequence form along the lines of Theorems 1 and 2 is not, however, convenient in this case.

Proof of Theorems 4 and 5 . We construct the proofs in parallel fashion as we did with Theorems 1 and 2 .

Suppose $X^{-}$has an analytic characteristic function. Take $\beta$ arbitrary with $0<\beta<\mu$. We consider to begin with

$$
\begin{aligned}
K_{n} & =\int_{n \beta}^{n \mu} e^{-s x} H_{n}(x) d x \\
& \leqq n e^{-n \beta s} \int_{\beta}^{\mu} H_{n}(n x) d x \\
& =n e^{-n \beta s}(\mu-\beta) H_{n}(n \xi)
\end{aligned}
$$

for some $\xi=\xi(n)$ in $\beta<\xi<\mu$. $(X-\xi)^{-}$has an analytic characteristic function and $E(X-\xi)>0$. It follows from Theorem B of [4] and the inequality 
$H_{m} \leqq F_{m}$ that for any $r$ in some interval $0<r<R$,

$$
\sum e^{r m} H_{m}(m \xi)<\infty,
$$

and hence, considering the totality of series (20) as $n$ varies,

$$
H_{n}(n \xi)=o\left(e^{-r n}\right) \text { as } n \rightarrow \infty .
$$

Therefore,

$$
\begin{aligned}
\sum_{n=1}^{\infty} e^{r n} K_{n} & \leqq(\mu-\beta) \sum_{n=1}^{\infty} n e^{-n \beta s} H_{n}(n \xi) e^{r n} \\
& <\infty \quad \text { as } s \rightarrow \mu^{-1} r+
\end{aligned}
$$

in view of $(21)$ and so

$$
\lim _{s \rightarrow \mu^{-1}{ }_{r+}}\left(s-\mu^{-1} r\right) \sum_{n=1}^{\infty} e^{r n} K_{n}=0 .
$$

Next consider

$$
L_{n}=\int_{n \mu}^{\infty} e^{-s x}\left\{1-H_{n}(x)\right\} d x \quad(s>0) .
$$

Using the mean value theorem and the fact that $H_{n}(n x) \rightarrow 1$ as $n \rightarrow \infty$ for $x>\mu$, we may write

$$
L_{n}=\delta_{n} \int_{n \mu}^{\infty} e^{-s x} d x=\delta_{n} s^{-1} e^{-n \mu s},
$$

where $\delta_{n}>0$ and $\delta_{n} \rightarrow 0$ as $n \rightarrow \infty$ uniformly in $s>0$. Now given arbitrary $\epsilon>0$ we can choose an integer $n_{0}(\epsilon)$ so large that $\delta_{n}<\epsilon$ for all $n>n_{0}$. Then, for $s>\mu^{-1} r$,

$$
\begin{aligned}
\sum_{n=1}^{\infty} e^{r n} L_{n} & =s^{-1} \sum_{n=1}^{\infty} \delta_{n} e^{-n \mu\left(s-\mu^{-1} r\right)} \\
& <s^{-1} \sum_{n=1}^{n_{0}} \delta_{n} e^{-n \mu\left(s-\mu^{-1}\right)}+\epsilon s^{-1} \sum_{n=1}^{\infty} e^{-n \mu\left(s-\mu^{-1} r\right)},
\end{aligned}
$$

and since for $s>\mu^{-1} r$,

$\sum_{n=1}^{\infty} e^{-n \mu\left(s-\mu^{-1} r\right)}=e^{-\mu\left(s-\mu^{-1} r\right)} /\left(1-e^{-\mu\left(s-\mu^{-1} r\right)}\right) \sim 1 / \mu\left(s-\mu^{-1} r\right) \quad$ as $\quad s \rightarrow \mu^{-1} r+$, it follows that

$$
\lim _{s \rightarrow \mu^{-1}{ }_{r+}}\left(s-\mu^{-1} r\right) \sum_{n=1}^{\infty} e^{r n} L_{n}=0,
$$

so that combining (22) and (23),

$$
\lim _{s \rightarrow \mu^{-}{ }^{-1}+}\left(s-\mu^{-1} r\right) \sum_{n=1}^{\infty} e^{r n}\left(L_{n}-K_{n}\right)=0 .
$$

Now consider the function

$$
\begin{aligned}
\Phi_{\beta}(x) & =\sum_{n=1}^{\infty} e^{r n} H_{n}(x) U(x-n \beta) \\
& =\sum_{n=1}^{\infty} e^{r n} U(x-n \mu)-\sum_{n=1}^{\infty} e^{r n}\left\{U(x-n \mu)-H_{n}(x)\right\} U(x-n \beta) .
\end{aligned}
$$

Taking Laplace transforms, we have

$$
\Phi_{\beta}{ }^{0}(s)=s^{-1} \sum_{n=1}^{\infty} e^{-n \mu\left(s-\mu^{-1} r\right)}-\sum_{n=1}^{\infty} e^{r n}\left(L_{n}-K_{n}\right),
$$


from which it follows, using (24), that $\left(s-\mu^{-1} r\right) \Phi_{\beta}{ }^{0}(s) \rightarrow r^{-1}$ as $s \rightarrow \mu^{-1} r+$. Now let

$$
\Theta_{\beta}{ }^{0}\left(s-\mu^{-1} r\right)=\Phi_{\beta}{ }^{0}(s) ;
$$

we have $w \Theta_{\beta}{ }^{0}(w) \rightarrow r^{-1}$ as $w \rightarrow 0+$, and appealing to a Tauberian theorem of Widder [7], Theorem 4.3, 192, we obtain

$$
t^{-1} \int_{0}^{t} \Theta_{\beta}(x) d x \rightarrow r^{-1} \text { as } t \rightarrow \infty
$$

Then, following through exactly the analysis that we used in the case of Theorems 1 and 2 (we are here dealing with the case $\gamma=0, L(t)=1, \alpha \mu^{-\gamma}[\Gamma(\gamma+2)]^{-1}=r^{-1}$ ) we find from (26) that

$$
\lim _{t \rightarrow \infty} \Theta_{\beta}(t)=r^{-1} \text {. }
$$

However, using (25) and the uniqueness theorem for Laplace transformations, $\Theta_{\beta}(t)=e^{-r \mu^{-1} t} \Phi_{\beta}(t)$, so that $(27)$ gives

$$
\lim _{t \rightarrow \infty} e^{-r \mu^{-1} t} \Phi_{\beta}(t)=r^{-1} .
$$

Now write

$$
\sum_{n=1}^{\infty} e^{r n} H_{n}(x)=\Phi_{\beta}(x)+\Psi_{\beta}(x)
$$

where

$$
\Psi_{\beta}(x)=\sum_{n=1}^{\infty} e^{r n} H_{n}(x)\{1-U(x-n \beta)\},
$$

$\sum e^{r n} H_{n}(x)$ being finite for $r$ in $0<r<R$ by (20). Also it is clear from (30) and (20) that for all $x, \Psi_{\beta}(x) \leqq \sum_{n=1}^{\infty} e^{r n} H_{n}(n \beta)<\infty$. We therefore have $e^{-r \mu^{-1} x} \Psi_{\beta}(x) \rightarrow 0$ as $x \rightarrow \infty$, and hence by $(28), \sum_{n=1}^{\infty} e^{r n} H_{n}(x) \sim r^{-1} e^{r \mu^{-1} x}$ as $x \rightarrow \infty$. This establishes the sufficiency parts of Theorems 4 and 5 .

The necessity parts of the theorems follow readily from Theorem B of [4]. We have $\sum e^{r n} H_{n}(x)<\infty$ for all $x,-\infty<x<\infty$ and Theorem $\mathrm{B}$ gives the result directly in the case $H_{n}(x)=F_{n}(x)$. In the case $H_{n}(x)=G_{n}(x)$, we take $x>0$ and make use of the well known relations $G_{n}(x)=\operatorname{Pr}\left(M_{n} \leqq x\right) \geqq \operatorname{Pr}\left(M_{n} \leqq 0\right)$ $\geqq n^{-1} \operatorname{Pr}\left(S_{n} \leqq 0\right)$. It is clear that the convergence of $\sum e^{r n} \operatorname{Pr}\left(M_{n} \leqq x\right)$ implies the convergence of $\sum n^{-1} e^{r n} \operatorname{Pr}\left(S_{n} \leqq 0\right)$ and hence that of $\sum e^{s n} \operatorname{Pr}\left(S_{n} \leqq 0\right)$ for $s<r$ and making use of Theorem B again we see that $X^{-}$must have an analytic characteristic function. This completes the proofs of both Theorems 4 and 5 .

3. Application to a first passage problem. Let $X_{i}, i=1,2,3, \cdots$ be independent and identically distributed random variables with $E|X|<\infty$, $E X=\mu>0$. Write $S_{n}=\sum_{i=1}^{n} X_{i}$ and $M_{n}=\max _{1 \leqq k \leqq n} S_{k}$. Consider a single boundary at $x(\geqq 0)$ so that if

$$
\begin{aligned}
G_{0}(x) & =1, \\
G_{n}(x) & =\operatorname{Pr}\left(M_{n} \leqq x\right), \quad n \geqq 1,
\end{aligned}
$$


the probability $p_{n}$ that the first passage time, $M(x)$, out of the interval $(-\infty, x]$ for the process $S_{n}$ is $n$ is given by $p_{n}=G_{n-1}(x)-G_{n}(x), n \geqq 1$.

We introduce the probability generating function $P(\lambda)=\sum_{r=1}^{\infty} \lambda^{r} p_{r}$ for the first passage time distribution $\operatorname{Pr}(M(x)=n)=p_{n}$.

Formally differentiating,

$$
P^{(1)}(1)=E[M(x)]=1+\sum_{r=1}^{\infty} G_{r}(x),
$$

and for $k>1$,

$$
\begin{aligned}
P^{(k)}(1) & \left.=(\alpha)_{k} \quad \text { (the } k \text { th factorial moment of } M(x)\right) \\
& =k \sum_{r=k-1}^{\infty}(r)_{k} G_{r}(x)=\sum_{r=0}^{k} s(k, r) E\left\{[M(x)]^{r}\right\},
\end{aligned}
$$

where $(r)_{k}=r(r-1)(r-2) \cdots(r-k+1)$ and $s(k, r)$ are the Stirling numbers of the first kind. It is thus clear that $E\left\{[M(x)]^{r}\right\}<\infty$ for some positive integer $r$ if and only if $\sum n^{r-1} G_{r}(x)<\infty$. Also, the random variable $M(x)$ has an analytic characteristic function if and only if the radius of convergence of $P(\lambda)$ is greater than unity or equivalently if $\sum e^{r n} G_{n}(x)<\infty$ for some $r>0$.

As a particular case of Theorem 2 we obtain for integral $k \geqq 1$,

$$
k x^{-k} \sum_{n=1}^{\infty} n^{k-1} G_{n}(x) \rightarrow \mu^{-k} \quad \text { as } \quad x \rightarrow \infty,
$$

so long as $E\left|X^{-}\right|^{k+1}<\infty$. Therefore, in view of the above comments, we see that as $x \rightarrow \infty, E\left\{\left[x^{-1} M(x)\right]^{r}\right\} \rightarrow \mu^{-r}$ for integral $r \geqq 1$ so long as $E\left|X^{-}\right|^{r+1}<\infty$. (The $r=1$ and $r=2$ cases are included in the results of [2] and [1] respectively). If $E\left|X^{-}\right|^{r}=\infty$ then Theorem 3 shows us that $\sum n^{r-1} G_{n}(x)$ diverges and hence that $E\left\{\left[x^{-1} M(x)\right]^{r}\right\}=\infty$. If we have the condition that $X^{-}$possesses an analytic characteristic function, then it follows from Theorem B of [4] and the inequality, $\operatorname{Pr}\left(S_{n} \leqq x\right) \geqq \operatorname{Pr}\left(M_{n} \leqq x\right)=G_{n}(x)$, that $M(x)$ possesses an analytic characteristic function. We have, in fact, for $r>0$ sufficiently small,

$$
\begin{aligned}
E\left[e^{r M(x)}\right] & =\sum_{n=1}^{\infty} e^{r n} P_{n} \\
& =e^{r}+\left(e^{r}-1\right) \sum_{n=1}^{\infty} e^{r n} G_{n}(x),
\end{aligned}
$$

so that by Theorem 5 ,

$$
E\left[e^{r M(x)}\right] \sim r^{-1}\left(e^{r}-1\right) e^{r^{-1}-1 x} \quad \text { as } \quad x \rightarrow \infty .
$$

The function $r^{-1}\left(e^{r}-1\right) e^{r \mu^{-1} x}$ is the Laplace-Stieltjes transform of the convolution of a rectangular distribution on the interval $(0,1)$ and a degenerate distribution at $x \mu^{-1}$. We have therefore obtained the following theorem:

Theorem 6. Suppose $E X=\mu>0$. If for some integral $r \geqq 1, E\left|X^{-}\right|^{r+1}<\infty$, then

$$
E\left\{\left[x^{-1} M(x)\right]^{r}\right\} \rightarrow \mu^{-r} \quad \text { as } \quad x \rightarrow \infty .
$$

If $X^{-}$possesses an analytic characteristic function then for $r>0$ sufficiently small

$$
E\left[e^{r M(x)}\right] \sim r^{-1}\left(e^{r}-1\right) e^{r \mu-1 x} \quad \text { as } \quad x \rightarrow \infty .
$$


One can further use a method due to Doob based on the strong law of large numbers to obtain the following result:

Theorem 7. Suppose $E X=\mu>0$. Then,

$$
x^{-1} M(x) \rightarrow \text { a.s. } \mu^{-1} \quad \text { as } \quad x \rightarrow \infty .
$$

Proof. According to the strong law of large numbers, we have for $0<\epsilon<\mu$ and sufficiently large $n,(\mu-\epsilon) n \leqq S_{n} \leqq(\mu+\epsilon) n$ with probability one. In particular, if $n=M(x)$ the left hand side implies $M(x) / x \leqq 1 /(\mu-\epsilon)$ and if $n=M(x)+1$ the right side implies $M(x)+1 \geqq x /(\mu+\epsilon)$. Thus, for large $x, 1 /(\mu+\epsilon)-1 / x \leqq M(x) / x \leqq 1 /(\mu-\epsilon)$ with probability one. The result follows.

Acknowledgments. I am indebted to J. F. Hannan for a helpful discussion on the subject of Lemma 1 and to Y. S. Chow for the observation which led to Theorem 7 .

\section{REFERENCES}

[1] Chow, Y. S. (1966). On the moments of some one-sided stopping rules. Ann. Math. Statist. 37 382-387.

[2] Chow, Y. S. and Roвbins, H. (1963). A renewal theorem for random variables which are dependent or non-identically distributed. Ann. Math. Statist. 34 390-395.

[3] Doetsch, G. (1950). Handbuch der Laplace-Transformation 1. Verlag-Birkhauser, Basel.

[4] Heyde, C. C. (1964). Two probability theorems and their application to some first passage problems. J. Austral. Math. Soc. 4 214-222.

[5] Loغ̀ve, M. (1960). Probability Theory (2nd edition). Van Nostrand, New York.

[6] Sмiтн, W. L. (1964). On the elementary renewal theorem for nonidentically distributed random variables. Pacific $J$. Math. 14 673-699.

[7] Widder, D. V. (1946). The Laplace Transform. Princeton Univ. Press. 